\title{
Genetic Polymorphisms in ADH1B and ALDH2 Are Associated with Colorectal Tumors in Japan: A Case-Control Study
}

\author{
Akiko Shiotani', Hideki Ishikawa2 ${ }^{2}$ Michihiro Mutoh ${ }^{3 *}$, Tatsuya Takeshita4, \\ Tomiyo Nakamura5, Kanehisa Morimoto6, Toshiyuki Sakai², Keiji Wakabayashi" \\ Nariaki Matsuura1 \\ ${ }^{1}$ Department of Molecular Pathology, Osaka University Graduate School of Medicine \& Health Science, Osaka, \\ Japan \\ ${ }^{2}$ Department of Molecular-Targeting Cancer Prevention, Kyoto Prefectural University of Medicine, Kyoto, Japan \\ ${ }^{3}$ Epidemiology and Prevention Division, Research Center for Cancer Prevention and Screening, National Cancer \\ Center, Tokyo, Japan \\ ${ }^{4}$ Department of Public Health, Wakayama Medical University School of Medicine, Wakayama, Japan \\ ${ }^{5}$ Department of Food Nutrition, Faculty of Agriculture, Ryukoku University, Shiga, Japan \\ ${ }^{6}$ Department of Hygiene and Preventive Medicine, Osaka University Graduate School of Medicine, \\ Osaka, Japan \\ ${ }^{7}$ Graduate Division of Nutritional and Environmental Sciences, University of Shizuoka, Shizuoka, Japan \\ Email: *mimutoh@ncc.go.jp
}

Received 25 September 2015; accepted 9 November 2015; published 12 November 2015

Copyright (C) 2015 by authors and Scientific Research Publishing Inc.

This work is licensed under the Creative Commons Attribution International License (CC BY). http://creativecommons.org/licenses/by/4.0/

c) (i) Open Access

\section{Abstract}

Background: Unlike with esophageal cancer, acetaldehyde levels and genetic polymorphisms in alcohol dehydrogenase have not yet been shown to be contributing factors for colorectal cancer (CRC). This study aimed to clarify the mechanism of CRC development related to alcohol consumption and to the presence of genetic polymorphisms in the alcohol dehydrogenase, ADH1B and aldehyde dehydrogenase, ALDH2. Methods: This was a case-control study (221 cases and 179 controls) in patients with adenomas and intramucosal tumors who underwent endoscopic removal of all tumors. The amount of alcohol consumption was determined using a self-recorded questionnaire, and the tumor information was obtained from colonoscopy results. Blood samples were taken to analyze the following polymorphisms: ALDH2 Glu504Lys and ADH1B His48Arg. Results: The polymorphisms in ADH1B and ALDH2 had little influence on the development of colorectal adenoma or intramucosal cancer. Patients with ALDH2 (Glu/Glu) were more tolerant of alcohol than those with ALDH2 (Glu/Lys and Lys/Lys). Next, we examined certain combinations of the ADH1B genotypes. In the ALDH2 (Glu/Glu) group, an increased risk (OR = 3.4; 95\% CI 1.4 - 8.4; P =

\footnotetext{
${ }^{*}$ Corresponding author.
}

How to cite this paper: Shiotani, A., Ishikawa, H., Mutoh, M., Takeshita, T., Nakamura, T., Morimoto, K., Sakai, T., Wakabayashi, K. and Matsuura, N. (2015) Genetic Polymorphisms in ADH1B and ALDH2 Are Associated with Colorectal Tumors in Japan: A Case-Control Study. Journal of Cancer Therapy, 6, 1054-1062. http://dx.doi.org/10.4236/jct.2015.612115 
0.009) was observed among moderate/heavy drinkers with ADH1B (His/His). In the ALDH2 (Glu/Lys and Lys/Lys) group, an increased risk (OR $=4.2 ; 95 \% \mathrm{CI} 1.1-16.7 ; \mathrm{P}=0.041$ ) was found among moderate/heavy drinkers with ADH1B (Arg/His and Arg/Arg). Conclusions: ADH1B and ALDH2 activity may be involved in the development of CRC.

\title{
Keywords
}

\author{
Genetic Polymorphisms, Colorectal Tumor, Alcohol Intake, Alcohol Dehydrogenase-1B, Aldehyde \\ Dehydrogenase-2
}

\section{Introduction}

Many epidemiological studies have confirmed that alcohol consumption is strongly associated with the development of colorectal cancer (CRC) [1]. Several epidemiological studies conducted in Japan have reported a link between alcohol consumption and CRC [2]-[4]. Because the incidence of CRC was increasing in Japan [5] along with the amount of alcohol consumption [6] at the time, this study was performed, drinking was considered to be strongly associated with the increased incidence of CRC in Japan.

The mechanisms of CRC development and progression related to alcohol consumption remain mostly unknown. With regard to esophageal cancer, the involvement of acetaldehyde, a metabolite of alcohol, has been implicated in the carcinogenesis of esophageal cancer based on studies of genetic polymorphisms in ALDH2 [7]-[11]. However, many published studies have reported that acetaldehyde is not a contributing factor for CRC [12]-[14].

Alcohol is oxidized to acetaldehyde mainly by ADH which consists of peptides coded by ADH1B and ADH1C. Acetaldehyde is further oxidized to acetic acid, mainly by low $\mathrm{Km}$ acetaldehyde dehydrogenases (such as ALDH2). Certain genetic polymorphisms in two representative alcohol-metabolizing genes, ADH1B and ALDH2, have been characterized regarding their role in defining the metabolizing capacity of these enzymes [15] [16]. Concerning the ADH1B Arg47His polymorphisms, ADH1B47His+ has greater activity than ADH1B Arg/Arg. With the ALDH2 504Lys polymorphisms, the 504Lys allele encodes an enzymatically inactive subunit. The activity level of ALDH2 in people with the ALDH2 Glu/Lys genotype is only $6 \%$ of that in people with the ALDH2 Glu/Glu genotype, and the activity level in people with the ALDH2Lys/Lys genotype is extremely low. The frequency of the ADH1B 47His and ALDH2 Glu504Lys alleles, both of which lead to high concentrations of acetaldehyde, is particularly high in East Asian populations. We have conducted a study combining the genetic polymorphisms that affect the enzymatic activity of these two proteins with the purpose of clarifying the mechanism of CRC development related to alcohol consumption.

This study was designed as a case-control study in patients with adenoma and intramucosal tumors to investigate the involvement of alcohol and its metabolites in the early stages of CRC.

\section{Methods}

\subsection{Subjects}

The subjects in this study were participants in a randomized clinical trial of the administration of a wheat bran and Lactobacillus casei preparation for the prevention of colorectal tumors. The details of this trial have previously been reported [17]. Briefly, the participants included 445 male and female patients aged 40 to 65 years who had undergone a total colonoscopy at Osaka Medical Center for Cancer and Cardiovascular Diseases, a hospital located in the city of Osaka, Japan, in which two or more colorectal tumors (early intramucosal tumors, adenomas) were identified; these patients subsequently underwent the endoscopic removal of all tumors. Subjects for whom the genetic polymorphisms of ADH1B and ALDH2 were not determined (214 subjects) and those with missing values in the correction items (10 subjects) were excluded, and the remaining 221 were included in the analysis. The participants were recruited from June 1993 to September 1997.

The controls in this study were selected from 186 men and women aged 40 to 65 years who underwent a 
complete medical checkup at the same hospital during the same time period. Among these patients, those with missing values in the correction items ( 7 controls) were excluded, and 179 were ultimately included in the analysis.

Patients with malignant diseases who underwent intestinal resections, except for appendectomy, as well as those with familial adenomatous polyposis or serious diseases were excluded from both groups.

\subsection{Ethical Approval}

This study was reviewed and approved by the Ethics Committee of Osaka Medical Center for Cancer and Cardiovascular Diseases. Written informed consent was obtained from all the participants in the Case Group and Control Group, and all clinical investigation had to be conducted according to the principles expressed in the Declaration of Helsinki. This study was performed from1993 to 2002, and registration of the clinical trial has not started. Thus, this study does not have a clinical trial number.

\subsection{Measurements}

After providing consent to participate in the study, the participants in the Case Group and Control Group were interviewed to obtain their height, weight, medical history, medication history, and family history as well as information on diet, drinking, smoking, lifestyle, physical activity, and the use of NSAIDs, such as aspirin. To obtain dietary information, a self-recorded questionnaire survey was administered using the dietary-recording method for three consecutive weekdays at the time of entry into the study. The participants visited the study site on the fourth day, and they were interviewed by a specialized nutritionist regarding their diet. Energy and nutrient intake was calculated using the nutrient calculation database program of Osaka Medical Center for Cancer and Cardiovascular Diseases. Blood samples were taken for genetic tests. Information about the grade (adenoma or intramucosal cancer), diameter, number and site of all of the tumors found in the Case Group was obtained from past colonoscopy results.

\subsection{Determining Drinking Behavior}

Alcohol intake at the time of entry into the study was obtained in terms of grams of alcohol. A self-recorded questionnaire was used to collect information on drinking behavior, and nutritionists clarified any unclear statements through interviews. In the self-recorded questionnaire, current drinking behavior was ascertained according to the following six grades: 1) drink every day, 2) drink approximately 3 - 5 times per week, 3) drink approximately 1 - 2 times per week, 4) drink approximately 1 - 3 times per month, 5) drink approximately 1 - 10 times per year, and 6) do not drink. In addition, those who replied that they drink were asked to state what types of liquor (Japanese sake, beer, Shochu, or whiskey) and how much (how many sake bottles, large beer bottles, or glasses) they drank to calculate the daily alcohol intake (in grams). In this study, we categorized the daily amount of alcohol intake into the following four groups: non-drinker, light drinker ( $<16 \mathrm{~g} /$ day), moderate drinker ( $\geq 16$ but $<35 \mathrm{~g}$ /day), and heavy drinker ( $\geq 35.0 \mathrm{~g}$ /day).

\subsection{Measurement of Genetic Polymorphisms}

Blood samples were taken from the participants. We analyzed the ALDH2Glu504Lys and ADH1B His48Arg polymorphisms. Each of the ADH1B and ALDH2 genotypes was determined based on a previously published method [18] [19]. For ALDH2, Glu/Glu was called "high", and Glu/Lys and Lys/Lys were called "low"; for ADH1B, Arg/His and Arg/Argwere called "slow", and His/His was called "fast".

\subsection{Analytical Methods}

Comparisons between the Case Group and Control Group with respect to sex, height, weight and body mass index (BMI) were analyzed by t-test. Comparisons between the Case Group and Control Group regarding the amount of alcohol intake, smoking and genotype were performed by calculating the odds ratio (OR) and 95\% confidence interval (CI). With regard to the polymorphisms and their combinations in each group, the OR adjusted for age/sex or for age/sex/BMI/smoking and the 95\% CI were calculated using a logistic regression model. IBM SPSS Statistics Ver. 20 was used for the statistical analyses. An unpaired t-test was used to compare the 
background factors between the two groups, and P values less than 0.05 were considered statistically significant.

\section{Results}

The characteristics of the participants in this study are shown in Table 1. There were more men than women in both the Case Group and the Control Group. With respect to the age of the participants, the Case Group tended to be slightly younger than the Control Group. There were almost no differences in terms of height, weight or BMI. The numbers of alcohol drinkers and smokers were significantly higher in the Case Group. The genotype frequencies of all of the tested genetic polymorphisms followed the Hardy-Weinberg equilibrium in the Control Group, and the allele frequencies nearly matched the frequencies that have previously been reported in Japan [20]. The Arg/Arg genotype of ADH1B and the Lys/Lys (low) genotype of ALDH2 were slightly less frequent in the Case Group.

Table 2 shows the classification of the ADH1B and ALDH2 polymorphisms, the classification of the combined polymorphisms, and the ORs for each. There were no distinctive findings related to the combination of these two genes.

Table 1. Characteristics of the study subjects.

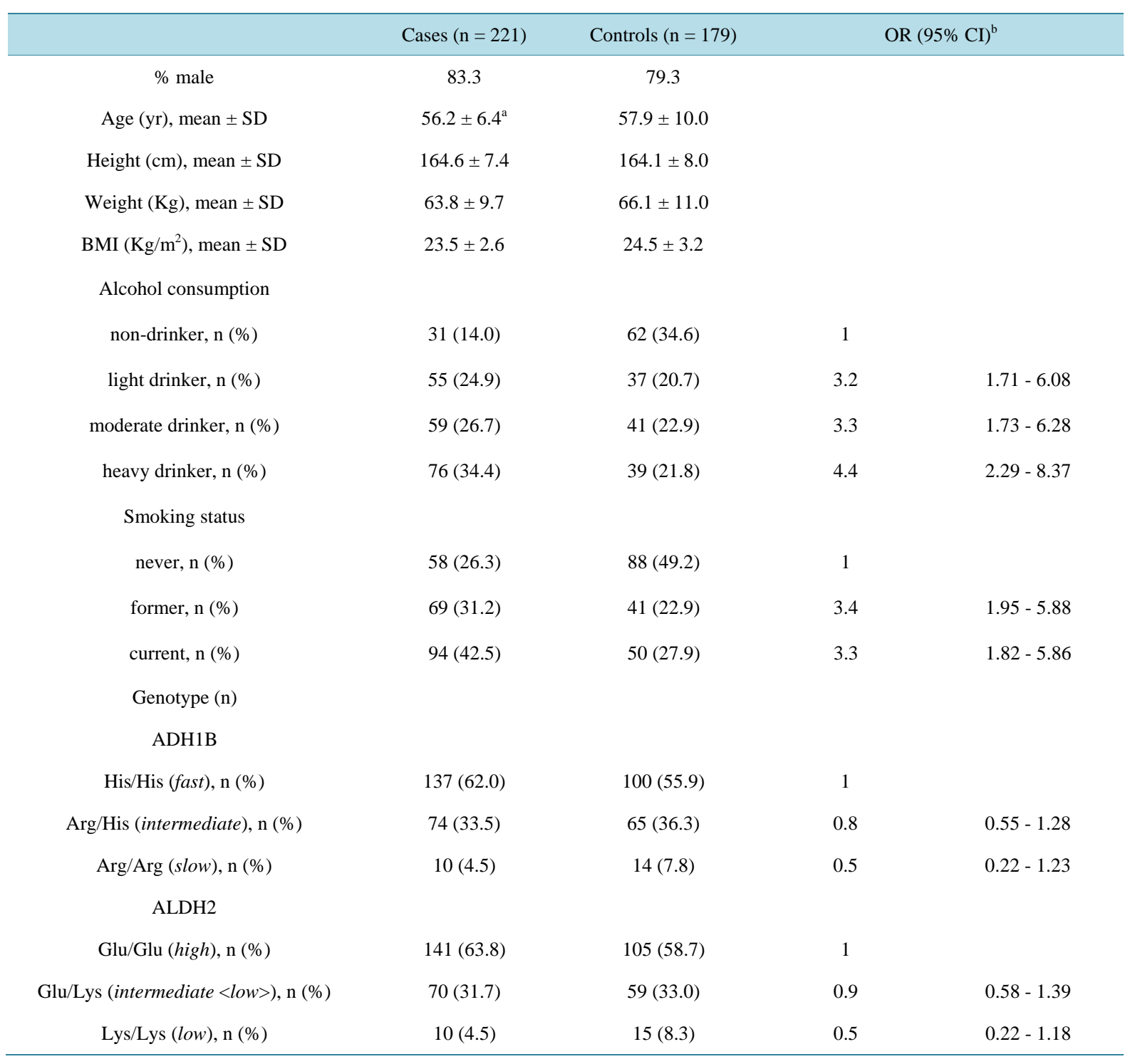

${ }^{\mathrm{a}}$ Significantly different from controls: $\mathrm{P}<0.05$; ${ }^{\mathrm{b}} \mathrm{OR}$ is adjusted by sex/age. 
Table 2. Odds ratios by ADH1B and ALDH2.

\begin{tabular}{|c|c|c|c|c|c|c|}
\hline & \multirow{2}{*}{ Cases } & \multirow{2}{*}{ Controls } & \multicolumn{2}{|c|}{ Model 1} & \multicolumn{2}{|c|}{ Model 2} \\
\hline & & & OR & $95 \%$ CI & OR & $95 \%$ CI \\
\hline ADH1B (fast) & 137 & 100 & 1 & & 1 & \\
\hline ADH1B (slow) & 84 & 79 & 0.8 & $0.52-1.17$ & 0.8 & $0.51-1.20$ \\
\hline ALDH2 (high) & 141 & 105 & 1 & & 1 & \\
\hline ALDH2 (low) & 80 & 74 & 0.8 & $0.55-1.23$ & 0.9 & $0.61-1.47$ \\
\hline ADH1B (fast)·ALDH2 (high) & 90 & 57 & 1 & & 1 & \\
\hline ADH1B (fast)·ALDH2 (low) & 47 & 43 & 0.7 & $0.42-1.21$ & 0.9 & $0.52-1.66$ \\
\hline ADH1B (slow)·ALDH2 (high) & 51 & 48 & 0.7 & $0.40-1.15$ & 0.8 & $0.45-1.33$ \\
\hline ADH1B (slow) ALDH2 (low) & 33 & 31 & 0.7 & $0.38-1.25$ & 0.7 & $0.40-1.41$ \\
\hline ADH1B (fast) & 137 & 100 & 1 & & 1 & \\
\hline
\end{tabular}

1. Adjusted by sex and age; 2. Adjusted by sex, age, BMI, smoking status and drinking.

Table 3 presents the ORs for the polymorphisms of these two genotypes based on alcohol intake. The OR did not increase according to alcohol intake in participants with ALDH2 (low). However, the ratio significantly increased to 7.2 in those with ALDH2 (high), even among light drinkers, suggesting that patients with ALDH2 (high) were more tolerant of alcohol than those with ALDH2 (low). The differences in the ORs for the polymorphisms in ADH1B based on alcohol intake were not as obvious as those for polymorphisms in ALDH2.

In the non-drinkers group, the OR for ADH1B (fast) compared to ADH1B (slow) was 1.94 (95\% CI: 0.66 5.68; P = 0.229), and the OR (95\% CI) for ALDH2 (high) compared to ALDH2 (low) was 0.40 (95\% CI: 0.14 $1.13 ; \mathrm{P}=0.396)$.

The ORs based on alcohol intake for the two gene polymorphisms in combination are shown in Table 4. In this analysis, the non-drinkers and light drinkers were analyzed as one group, and the moderate and heavy drinkers were analyzed as one group.

For patients with ALDH2 (high), an increased risk (OR = 3.4; 95\% CI: 1.4 - 8.4; P = 0.009) was observed among moderate/heavy drinkers with ADH1B (fast), whereas no increase in risk was observed among moderate/heavy drinkers with ADH1B (slow) (Table 4). In the analysis in which the interaction between the ADH1B polymorphisms and moderate/heavy alcohol intake was added to the explanatory variables, the interaction was significant $(\mathrm{OR}=3.5 ; 95 \% \mathrm{CI}: 1.1-10.8 ; \mathrm{P}=0.029)$.

However, among patients with ALDH2 (low), an increased risk (OR = 4.2; 95\% CI: 1.1 - 16.7; P = 0.041) was found among moderate/heavy drinkers with ADH1B (slow), whereas no such increase was observed among moderate/heavy drinkers with ADH1B (fast) (Table 4). In the analysis in which the interaction between the ADH1B polymorphisms and moderate/heavy alcohol intake was added to the explanatory variables, the interaction was significant $(\mathrm{OR}=4.8$; 95\% CI: $1.0-22.1 ; \mathrm{P}=0.043)$.

\section{Discussion}

The results of this study suggest that the polymorphisms in ADH1B and ALDH2 have some influence on the development of colorectal adenoma or intramucosal cancer.

Although the relationship between alcohol consumption and CRC has almost certainly been confirmed by a number of epidemiological studies [21], the mechanism underlying this association remains unclear. Because the ALDH2 (low) genetic polymorphism increases the concentration of acetaldehyde after alcohol intake, individuals with this genotype are unable to drink much alcohol. This genotype is frequently found in Asian populations [22]. Acetaldehyde is strongly associated with the development of esophageal squamous cell carcinoma, which is a prevalent disease in Asian populations [23] [24]. However, because the relationship between alcohol intake and the development of CRC is observed regardless of race [1], it has been assumed from an epidemiological 
Table 3. Odds ratios for ADH1B and ALDH categorized by the amount of alcohol intake.

\begin{tabular}{|c|c|c|c|c|c|}
\hline & & non & light & moderate & heavy \\
\hline \multicolumn{6}{|c|}{ ADH1B } \\
\hline \multirow[t]{2}{*}{ fast } & $N$ (cases/controls) & $22 / 33$ & $31 / 22$ & $35 / 23$ & $49 / 22$ \\
\hline & OR (95\% CI) & 1 (REF) & $2.0(0.82-4.69)$ & $2.4(0.96-5.85)$ & $3.3(1.32-8.18)$ \\
\hline \multirow[t]{2}{*}{ slow } & $N$ (cases/controls) & $9 / 29$ & $24 / 15$ & $24 / 18$ & $27 / 17$ \\
\hline & OR (95\% CI) & 1 (REF) & $4.7(1.58-14.18)$ & $3.8(1.27$ - 11.63) & $3.3(1.06-10.27)$ \\
\hline \multicolumn{6}{|c|}{ ALDH2 } \\
\hline \multirow[t]{2}{*}{ high } & $N$ (cases/controls) & $11 / 34$ & $30 / 14$ & $37 / 26$ & $63 / 31$ \\
\hline & OR (95\% CI) & 1 (REF) & $7.2(2.64-19.45)$ & $4.6(1.78-12.04)$ & $6.2(2.39-16.07)$ \\
\hline \multirow[t]{2}{*}{ low } & $N$ (cases/controls) & $20 / 28$ & $25 / 23$ & $22 / 15$ & $13 / 8$ \\
\hline & OR (95\% CI) & 1 (REF) & $1.2(0.43-3.12)$ & $1.9(0.65-5.56)$ & $1.4(0.40-5.10)$ \\
\hline
\end{tabular}

OR is adjusted by sex, age, BMI and smoking status.

Table 4. Odds ratios for the combinations of ALDH2 and ADH1B categorized by the amount of alcohol intake.

\begin{tabular}{|c|c|c|c|}
\hline \multicolumn{4}{|c|}{ Odds ratio (95\% CI) of Moderate/Heavy drinkers to Non/Light drinkers } \\
\hline & & Non and light drinker & Moderate and heavy drinker \\
\hline \multicolumn{4}{|l|}{ ALDH2 (high) } \\
\hline \multirow[t]{3}{*}{ ADH1B (fast) } & $\mathrm{N}$ (cases/controls) & $19 / 26$ & $71 / 31$ \\
\hline & crude OR & 1 & $3.1(1.52-6.48)$ \\
\hline & adjusted $\mathrm{OR}^{\mathrm{a}}$ & 1 & $3.4(1.36-8.41)$ \\
\hline \multirow[t]{3}{*}{ ADH1B (slow) } & N (cases/controls) & $22 / 22$ & $29 / 26$ \\
\hline & crude OR & 1 & $1.1(0.51-2.47)$ \\
\hline & adjusted $\mathrm{OR}^{\mathrm{a}}$ & 1 & $0.8(0.31-1.92)$ \\
\hline \multicolumn{4}{|l|}{ ALDH2 (low) } \\
\hline \multirow[t]{3}{*}{ ADH1B (fast) } & $\mathrm{N}$ (cases/controls) & $34 / 29$ & $13 / 14$ \\
\hline & crude OR & 1 & $0.8(0.32-1.95)$ \\
\hline & adjusted $\mathrm{OR}^{\mathrm{a}}$ & 1 & $0.8(0.30-2.39)$ \\
\hline \multirow[t]{3}{*}{ ADH1B (slow) } & N (cases/controls) & $11 / 22$ & $22 / 9$ \\
\hline & crude OR & 1 & $4.9(1.69-14.12)$ \\
\hline & adjusted $\mathrm{OR}^{\mathrm{a}}$ & 1 & $4.2(1.06-16.66)$ \\
\hline
\end{tabular}

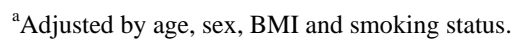

perspective that acetaldehyde is not closely related to the development of CRC. Actually, several papers have examined the relationship between CRC and the ALDH2 (low) polymorphism and found no obvious relationship between these two variables [12]-[14]. However, in population-based samples, it has been reported that the ALDH2 (low) polymorphism is associated with a reduced risk of CRC [14]. In our study, the OR did not increase according to alcohol intake among participants with ALDH2 (low). This result is markedly different from that previously reported in studies on esophageal cancer [7]-[11]. Based on this fact, there must be a major difference in the degree of acetaldehyde involvement in the development of colorectal cancer versus esophageal cancer. 
Because it is obvious that alcohol consumption increases the risk of CRC, we conducted a further examination of certain combinations involving the ADH1B genotypes. Relatively few studies have investigated the influence of alcohol intake on the combinations of these two alcohol metabolism-related polymorphisms [12] [16] [25][29]. With respect to esophageal cancer, a significantly increased risk was found among moderate drinkers with a combination of the ALDH2 Glu/Lys or ALDH2 Lys/Lys and ADH1B Arg/Arg and ADH1B Arg/His genotypes [25]. A significant interaction was also found among moderate drinkers with the ADH1B Arg/Arg and ALDH2 Glu/Lys combination [26]. The only data on CRC that we were able to confirm was that in the report by Matsuo et al. [27]. This report is a case-control study with 257 patients with CRC and 771 controls without cancer. In this report, the sex- and age-adjusted OR based on ADH1B His/His (fast) was 1.39 (1.02 - 1.87) for participants with ADH1B Arg/His, whereas the OR was 1.92 (1.06 - 3.46) for those with the Arg/Arg genotype, suggesting a significant risk increase. In our study, an interaction with moderate or high alcohol consumption was observed among participants with the combination of ALDH2 Glu/Glu and ADH1B His/His or with the combination of ALDH2 Lys+ and ADH1B Arg+. The combination of ALDH2 Glu/Glu and ADH1B His/His was reported by Matsuo et al. to be associated with an increased risk [28]. It has been also reported that the OR was 3.42 (0.57 - 20.38) when compared the subjects having ALDH2 Lys+ and ADH1B His/His with subjects having ALDH2 Glu/Glu and ADH1B His/His [14]. Although the mechanism by which the risk increases is unclear, this result is interesting. It is difficult to explain the mechanism of the interaction between the combination of ALDH2 Lys+ and ADH1B Arg+ and alcohol consumption. Because the CI of the OR was wide, further investigation will be needed.

In a recent study, the slope of the increase in blood acetaldehyde concentration was reported to be steeper in people with the ALDH2 Glu/Lys (low) and ADH1B Arg+ (slow) combination than in those with other combinations [29]. This suggests that the cancer risk may be increased because the first stage of alcohol metabolism is slower in these people, who therefore generate acetaldehyde for a longer period. In addition, Yokoyama et al. reported that alcoholic patients with the ADH1BHis/His (fast) genotype have a higher risk of developing complications, such as cirrhosis, pancreatitis, and diabetes [30]. These findings suggest that ADH1B activity has some influence on the living body. The limitations of the study include the retrospective design and a relatively small case number that might have resulted in difficulty to build a hypothesis for the mechanism of colorectal tumor development related to alcohol consumption. However, our results suggested that ADH1B and ALDH2 activity influence the development of CRC.

\section{Conclusion}

The activities of alcohol dehydrogenases may be involved in the development of colorectal tumors.

\section{Acknowledgements}

We express our sincere gratitude to Dr. Xing Yang for his assistance with the genetic testing in this study. This study was conducted with a Grant-in-Aid from the Third Term Comprehensive Control Research for Cancer, Health and Labor Sciences Research Grants of the Ministry of Health, Labour and Welfare (H22-3Term-General014), and from the Japan Agency for Medical Research and Development, AMED (15ck0106102h0102). The funders had no role in study design, data collection and analysis, decision to publish, or preparation of the manuscript.

\section{References}

[1] (2007) World Cancer Research Fund and American Institute for Cancer Research: American Institute for Cancer Research.

[2] Otani, T., Iwasaki, M., Yamamoyo, S., et al. (2003) Alcohol Consumption, Smoking, and Subsequent Risk of Colorectal Cancer in Middle-aged and Elderly Japanese Men and Women: Japan Public Health Center-based Prospective Study. Cancer Epidemiology Biomarkers \& Prevention, 12, 1492-1500.

[3] Shimizu, N., Nagata, C., Kametani, M., et al. (2003) Height, Weight, and Alcohol Consumption in Relation to the Risk of Colorectal Cancer in Japan: a Prospective Study. British Journal of Cancer, 88, 1038-1043. http://dx.doi.org/10.1038/sj.bjc.6600845

[4] Wakai, K., Kojima, M., Tamakoshi, K., et al. (2005) Alcohol Consumption and Colorectal Cancer Risk: Findings from the JACC Study. Journal of Epidemiology, 15, S173-179. 
[5] Yiu, H.Y., Whittemore, A.S. and Shibata, A. (2004) Increasing Colorectal Cancer Incidence Rates in Japan. International Journal of Cancer, 109, 777-781. http://dx.doi.org/10.1002/ijc.20030

[6] Higuchi, S., Matsushita, S., Maesato, H., et al. (2007) Japan: Alcohol Today. Addiction, 102, 1849-1862. http://dx.doi.org/10.1111/j.1360-0443.2007.01902.x

[7] Yokoyama, A., Kato, H., Yokoyama, T., et al. (2002) Genetic Polymorphisms of Alcohol and Aldehyde Dehydrogenases and Glutathione S-Transferase M1 and Drinking, Smoking, and Diet in Japanese Men with Esophageal Squamous Cell Carcinoma. Carcinogenesis, 23, 1851-1859. http://dx.doi.org/10.1093/carcin/23.11.1851

[8] Yang, C.X., Matsuo, K., Ito, H., et al. (2005) Esophageal Cancer Risk by ALDH2 and ADH2 Polymorphisms and Alcohol Consumption: Exploration of Gene-Environment and Gene-Gene Interactions. Asian Pacific Journal of Cancer Prevention, 6, 256-262.

[9] Chen, Y.J., Chen, C., Wu, D.C., et al. (2006) Interactive Effects of Lifetime Alcohol Consumption and Alcohol and Aldehyde Dehydrogenase Polymorphisms on Esophageal Cancer Risks. International Journal of Cancer, 119, 28272831. http://dx.doi.org/10.1002/ijc.22199

[10] Yang, S.J., Wang, H.Y., Li, X.Q., et al. (2007) Genetic Polymorphisms of ADH2 and ALDH2 Association with Esophageal Cancer Risk in Southwest China. World J Gastroenterol, 13, 5760-5764. http://dx.doi.org/10.3748/wjg.v13.i43.5760

[11] Matsuo, K., Hamajima, N., Shinoda, M., et al. (2001) Gene-Environment Interaction between an Aldehyde Dehydrogenase-2 (ALDH2) Polymorphism and Alcohol Consumption for the Risk of Esophageal Cancer. Carcinogenesis, 22, 913-916. http://dx.doi.org/10.1093/carcin/22.6.913

[12] Yin, G., Kono, S., Toyomura, K., et al. (2007) Alcohol Dehydrogenase and Aldehyde Dehydrogenase Polymorphisms and Colorectal Cancer: The Fukuoka Colorectal Cancer Study. Cancer Science, 98, 1248-1253. http://dx.doi.org/10.1111/j.1349-7006.2007.00519.x

[13] Matsuo, K., Hamajima, N., Hirai, T., et al. (2002) Aldehyde Dehydrogenase 2 (ALDH2) Genotype Affects Rectal Cancer Susceptibility Due to Alcohol Consumption. Journal of Epidemiology, 12, 70-76. http://doi.org/10.2188/jea.12.70

[14] Chen, B., Hu, K.W., Zhang, J.W., et al. (2015) A Critical Analysis of the Relationship between Aldehyde Dehydrogenases-2 Glu487Lys Polymorphism and Colorectal Cancer Susceptibility. Pathology \& Oncology Research, 21, 727733. http://dx.doi.org/10.1007/s12253-014-9881-8

[15] Matsuo, K., Wakai, K., Hirose, K., et al. (2006) Alcohol Dehydrogenase 2 His47Arg Polymorphism Influences Drinking Habit Independently of Aldehyde Dehydrogenase 2 Glu487Lys Polymorphism: Analysis of 2,299 Japanese Subjects. Cancer Epidemiology, Biomarkers \& Prevention, 15, 1009-1013. http://dx.doi.org/10.1158/1055-9965.EPI-05-0911

[16] Yokoyama, A., Muramatsu, T., Omori, T., et al. (2001) Alcohol and Aldehyde Dehydrogenase Gene Polymorphisms and Oropharyngolaryngeal, Esophageal and Stomach Cancers in Japanese Alcoholics. Carcinogenesis, 22, 433-439. http://dx.doi.org/10.1093/carcin/22.3.433

[17] Ishikawa, H., Akedo, I., Otani, T., et al. (2005) Randomized Trial of Dietary Fiber and Lactobacillus Casei Administration for Prevention of Colorectal Tumors. International Journal of Cancer, 116, 762-767. http://dx.doi.org/10.1002/ijc.21115

[18] Takeshita, T., Morimoto, K., Mao, X., et al. (1994) Characterization of the Three Genotypes of Low Km Aldehyde Dehydrogenase in a Japanese Population. Human Genetics, 94, 217-223.

[19] Takeshita, T., Mao, X.Q. and Morimoto, K. (1996) The Contribution of Polymorphism in the Alcohol Dehydrogenase Beta Subunit to Alcohol Sensitivity in a Japanese Population. Human Genetics, 97, 409-413.

[20] Yoshimura, K., Hanaoka, T., Ohnami, S., et al. (2003) Allele Frequencies of Single Nucleotide Polymorphisms (SNPs) in 40 Candidate Genes for Gene-Environment Studies on Cancer: Data from Population-Based Japanese Random Samples. Journal of Human Genetics, 48, 654-658. http://dx.doi.org/10.1007/s10038-003-0096-1

[21] Druesne-Pecollo, N., Tehard, B., Mallet, Y., et al. (2009) Alcohol and Genetic Polymorphisms: Effect on Risk of Alcohol-Related Cancer. The Lancet Oncology, 10, 173-180. http://dx.doi.org/10.1016/S1470-2045(09)70019-1

[22] Crabb, D.W., Edenberg, H.J., Bosron, W.F., et al. (1989) Genotypes for Aldehyde Dehydrogenase Deficiency and Alcohol Sensitivity. The Inactive ALDH2(2) Allele Is Dominant. Journal of Clinical Investigation, 83, 314-316. http://dx.doi.org/10.1172/JCI113875

[23] Pennathur, A., Gibson, M.K., Jobe, B.A., et al. (2013) Oesophageal Carcinoma. The Lancet, 381, 400-412. http://dx.doi.org/10.1016/S0140-6736(12)60643-6

[24] Ozawa, S., Tachimori, Y., Baba, H., et al. (2010) Comprehensive Registry of Esophageal Cancer in Japan, 2002. Esophagus, 7, 7-22. http://dx.doi.org/10.1007/s10388-010-0228-6 
[25] Yang, S.J., Wang, H.Y., Li, X.Q., et al. (2007) Genetic Polymorphisms of ADH2 and ALDH2 Association with Esophageal Cancer Risk in Southwest China. World Journal of Gastroenterology, 13, 5760-5764. http://dx.doi.org/10.3748/wjg.v13.i43.5760

[26] Wu, C.F., Wu, D.C., Hsu, H.K., et al. (2005) Relationship between Genetic Polymorphisms of Alcohol and Aldehyde Dehydrogenases and Esophageal Squamous Cell Carcinoma Risk in Males. World Journal of Gastroenterology, 11, 5103-5108. http://dx.doi.org/10.3748/wjg.v11.i33.5103

[27] Matsuo, K., Wakai, K., Hirose, K., et al. (2006) A Gene-Gene Interaction between ALDH2 Glu487Lys and ADH2 His47Arg Polymorphisms Regarding the Risk of Colorectal Cancer in Japan. Carcinogenesis, 27, 1018-1023. http://dx.doi.org/10.1093/carcin/bgi282

[28] Matsuo, K., Ito, H., Wakai, K., et al. (2005) One-Carbon Metabolism Related Gene Polymorphisms Interact with Alcohol Drinking to Influence the Risk of Colorectal Cancer in Japan. Carcinogenesis, 26, 2164-2171. http://dx.doi.org/10.1093/carcin/bgi196

[29] Yokoyama, A., Tsutsumi, E., Imazeki, H., et al. (2010) Polymorphisms of Alcohol Dehydrogenase-1B and Aldehyde Dehydrogenase-2 and the Blood and Salivary Ethanol and Acetaldehyde Concentrations of Japanese Alcoholic Men. Alcoholism: Clinical and Experimental Research, 34, 1246-1256. http://dx.doi.org/10.1111/j.1530-0277.2010.01202.x

[30] Yokoyama, A., Mizukami, T., Matsui, T., et al. (2013) Genetic Polymorphisms of Alcohol Dehydrogenase-1B and Aldehyde Dehydrogenase-2 and Liver Cirrhosis, Chronic Calcific Pancreatitis, Diabetes Mellitus, and Hypertension among Japanese Alcoholic Men. Alcoholism: Clinical and Experimental Research, 37, 1391-1401. http://dx.doi.org/10.1111/acer.12108 